\title{
THE DETECTION OF CHICORY IN DECOCTIONS OF CHICORY AND COFFEE.
}

CHARLES H. LAWALL AND LEROY FORMAN.

In December, 1913, the American Journal of Pharmacy contained an article by the above authors, in which it was proposed to differentiate between pure coffee decoctions and those which contain chicory or any other substance high in reducing-sugars, by taking into account the ratio of reducing-sugar to extractive. In confirmation of the success of the method we would say that in about twentyfive suits which were instituted for violation of the food laws, in which this method of analysis was applied to the samples, no instance is on record of a denial of the adulteration, and fines were immediately paid by nearly all of the offenders, among whom were included several high-class restaurants and hotels. The object of this peper is to confirm the conclusions given in the former article, and to submit some data upon the analyses of additional products and to offer some suggestions so as to ensure accuracy of judgment for those who desire to make it the basis of legal procedure.

In the first place, it must be understood that the strength of the coffee or the method of its preparation has little or no influence upon the results. This was not made as clear in the former article, as it might have been, so we will now state that, using the same coffee and employing such widely divergent methods of preparation as decoction, filtration and percolation, the ratio of reducing-sugars to extractive, did not exceed the maximum of $3 \%$ reducing-sugars calculated to extractive, as the following results will show:

Coffee prepared by filtration (simply pouring boiling water through the powdered material contained in a straining cloth) $\ldots \ldots \ldots \ldots \ldots \ldots \ldots$

Coffee prepared by decoction (powdered coffee boiled with water and filtered washing the residue on filter with boiling water) $\ldots \ldots \ldots \ldots \ldots \ldots \ldots$

Coffee prepared by percolation (ground coffee extracted in one of the common household perco-

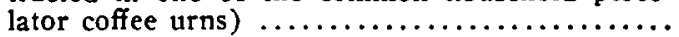

Percentage of Reducing-Sugars in Extractive

2.22

2.68

In the second place, it must be remembered that coffee-decostions, in common with other aqueous solutions containing organic matter, undergo decomposition quite readily, and as such decomposition is always accompanied by a loss of reducing-sugars, it is imperative that the analysis of the samples be made promptly after the sample is taken, or that some non-reducing preservative substance, like thymol, be added when the samples are taken or received, in order that the composition may remain constant until the analysis is made.

It must also be remembered that the addition of either sugar, or milk, or cream, vitiates the results, in so far as an accurate conclusion may be drawn. Canesugar, as commonly added for sweetening, has no influence upon the presence of 
reducing-sugars, but, needlessly, complicates the analysis, by rendering it imperative that the sucrose be separately estimated and subtracted from the extractive found, in order to arrive at a knowledge of the amount of extractive due to the coffee, upon which the ratio is based. The presence of milk or cream entirely vitiates the results, as milk-sugar is a reducing-sugar and as the presence of a small amount of milk or cream might escape detection, it is suggested that the centrifuging of about $10 \mathrm{cc}$. in a centrifuge such as is used for urinary sediments, or of a larger amount in a Babcock milk-testing machine, will quickly result in the identification and rejection of such samples that have had milk or cream added to them either accidentally or intentionally.

It has been found by us in laboratory practice applied to commercial samples, that about one-fourth of the adulterated samples contain a cereal in addition to the chicory. The detection of the cereal is easily made, easy by testing for starch (which is entirely absent in the beverage made from pure coffee) by taking $10 \mathrm{cc}$. of the liquid, acidulating with sulphuric acid and adding potassium permanganate solution, drop by drop, until the liquid is just decolorized, when a drop of iodine test-solution added to the filtrate gives the clear-blue starch reaction, which is ordinarily obscured by the dark color of the liquid.

The percentage of reducing-sugars in the extractive of a number of commercial products, is herewith given, as being of an informative character:

\begin{tabular}{|c|c|}
\hline 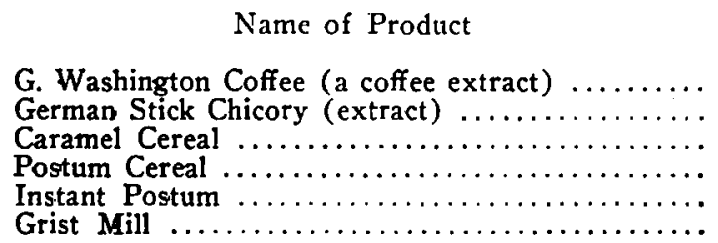 & $\begin{array}{l}\text { Percentage of Reducing-Sugars in } \\
\text { Extractive } \\
\text { less than } 1 \% \\
22.10 \\
13.69 \\
5.43 \\
6.36 \\
6.64\end{array}$ \\
\hline
\end{tabular}

The four last named are proprietary substitutes for coffee. All of them contained cereal, as shown by the starch-test above described.

\section{COMPARISON OF THE PHYSICAL PROPERTIES OF SOME OFFICIAL VOLATILE OILS WITH SPECIAL REFERENCE TO THE REQUIREMENTS OF THE U. S. P., 1910.}

FRANK RABAK.

Chemical Biologist, Drug-Plant and Poisonous-Plant Investigations. Bureau of Plant Industry.

The ninth decennial revision of the United States Pharmacopœia has resulted in numerous changes in the section devoted to volatile oils, especially with regard to the physical properties of the various oils. Among the properties described are color, odor, taste, specific gravity, optical rotation, and solubility, the refractive index being mentioned in only few instances. The importance of these properties should not be underestimated, as the varying quality of any volatile oil may be readily observed by means of them. Because of the inability of the 